\title{
Stochastic simulations of DNA in flow: Dynamics and the effects of hydrodynamic interactions
}

\author{
Richard M. Jendrejack, Juan J. de Pablo, and Michael D. Graham \\ Department of Chemical Engineering and Rheology Research Center, University of Wisconsin-Madison, \\ Madison, Wisconsin 53706-1691
}

(Received 15 May 2001; accepted 8 February 2002)

\begin{abstract}
We present a fully parametrized bead-spring chain model for stained $\lambda$-phage DNA. The model accounts for the finite extensibility of the molecule, excluded volume effects, and fluctuating hydrodynamic interactions (HI). Parameters are determined from equilibrium experimental data for $21 \mu \mathrm{m}$ stained $\lambda$-phage DNA, and are shown to quantitatively predict the non-equilibrium behavior of the molecule. The model is then used to predict the equilibrium and nonequilibrium behavior of DNA molecules up to $126 \mu \mathrm{m}$. In particular, the HI model gives results that are in quantitative agreement with experimental diffusivity data over a wide range of molecular weights. When the bead friction coefficient is fit to the experimental relaxation time at a particular molecular weight, the stretch in shear and extensional flows is adequately predicted by either a free-draining or HI model at that molecular weight, although the fitted bead friction coefficients for the two models differ significantly. In shear flow, we find two regimes at high shear rate $(\dot{\gamma})$ that follow different scaling behavior. In the first, the viscosity and first normal stress coefficient scale roughly as $\dot{\gamma}^{-6 / 11}$ and $\dot{\gamma}^{-14 / 11}$, respectively. At higher shear rates, these become $\dot{\gamma}^{-2 / 3}$ and $\dot{\gamma}^{-4 / 3}$. These regimes are found for both free-draining and HI models and can be understood based on scaling arguments for the diffusion of chain ends. (C) 2002 American Institute of Physics.
\end{abstract}

[DOI: $10.1063 / 1.1466831]$

\section{INTRODUCTION}

The ability to directly visualize individual DNA molecules via fluorescent staining allows one to obtain experimental data on the behavior of individual polymer molecules. ${ }^{1-8}$ Recent work ${ }^{9-11}$ has shown that simple beadspring or bead-rod models can reproduce some of the nonequilibrium properties of $\lambda$-phage DNA. That work has also demonstrated that, provided the friction coefficient is determined from the experimental relaxation time, these simple models can reproduce some properties of nondilute solutions. ${ }^{11}$ As important as that work has been, the models employed in these references have not included excluded volume effects or hydrodynamic interactions, and therefore cannot be used as predictive tools for molecules of differing sizes. Furthermore, experimental evidence ${ }^{3}$ indicates nonfree-draining behavior for DNA molecules as short as $3 \mu \mathrm{m}$. Recent studies ${ }^{12-14}$ have considered both excluded volume and hydrodynamic interactions in stochastic simulations of DNA. However, that work only considered chains of less than a micron in length and simulation times of less than a second. For simulations of transport in microfluidic devices which involve external fields, such as those proposed for DNA separation, ${ }^{1,15}$ a sound treatment of hydrodynamic interactions is essential.

In this work we present a fully parametrized beadspring model for DNA which includes finite extensibility, excluded volume effects and hydrodynamic interactions. The parameters in the model are inferred from available experimental data for $21 \mu \mathrm{m} \lambda$-phage DNA. The model satisfies all the molecular weight scaling laws for dilute solutions of lin- ear polymers, and therefore is expected to provide a useful predictive tool at higher molecular weights.

In Sec. II, we present the model and governing equations. In Sec. III, we define properties of interest in this paper, and, in Sec. IV, we present details of the simulation and parameter selection. In Sec. V A, we validate our model by quantitative comparison to available experimental data, and in Sec. V B we use the model to predict the behavior of DNA molecules up to $126 \mu \mathrm{m}$ in length. High shear rate scaling results are presented in Sec. VC for $84 \mu \mathrm{m}$ chains. In Sec. $\mathrm{V} \mathrm{D}$, we examine the dependence of the model on the degree of molecular discretization (number of Kuhn segments per spring). We conclude in Sec. VI with a summary of our results.

\section{MODEL}

We consider here a linear molecule represented by $N_{b}$ beads connected by $N_{s}=N_{b}-1$ springs. The model described here does not contain any mechanism for helical structuring or other sub-Kuhn length phenomena. It does, however, contain the most relevant physics for rheological properties and conformational properties which occur on longer length scales (gyration tensor and diffusivity tensor, for example). The governing stochastic differential equation for an isolated bead-spring chain immersed in a thermal solvent is given by ${ }^{16}$

$$
\begin{aligned}
& d \mathbf{R}=\left[\mathbf{K} \cdot \mathbf{R}+\frac{1}{k_{B} T} \mathbf{D} \cdot \mathbf{F}+\frac{\partial}{\partial \mathbf{R}} \cdot \mathbf{D}\right] d t+\sqrt{2} \mathbf{B} \cdot d \mathbf{W}, \\
& \mathbf{D}=\mathbf{B} \cdot \mathbf{B}^{T} .
\end{aligned}
$$


Here $k_{B}$ is Boltzmann's constant and $T$ is the absolute temperature. The vector $\mathbf{R}$ contains the $3 N_{b}$ spatial coordinates of the beads that constitute the polymer chain, $\mathbf{D}$ is a $3 N_{b}$ $\times 3 N_{b}$ diffusion tensor, and $\mathbf{F}$ is a force vector of length $3 N_{b}$. The $3 N_{b} \times 3 N_{b}$ matrix $\mathbf{K}$ is block diagonal with diagonal components $(\boldsymbol{\nabla} \mathbf{v})^{T}$, with $\mathbf{v}$ being the unperturbed solvent velocity. The components of $d \mathbf{W}$ are obtained from a real-valued Gaussian distribution with mean zero and variance $d t$.

Hydrodynamic interactions enter Eq. (1) through the offdiagonal components of the diffusion tensor. In this work, we take $\mathbf{D}$ to be the Rotne-Prager-Yamakawa (RPY) tensor, ${ }^{17}$ which can be written as

$\mathbf{D}_{i i}=\frac{k_{B} T}{\zeta} \mathbf{I}$

$\mathbf{D}_{i j}=\frac{k_{B} T}{8 \pi \eta R_{i j}}\left[C_{1} \mathbf{I}+C_{2} \frac{\mathbf{R}_{i j} \mathbf{R}_{i j}}{R_{i j}^{2}}\right] \quad$ if $i \neq j$ and $R_{i j} \geqslant 2 a$,

$\mathbf{D}_{i j}=\frac{k_{B} T}{\zeta}\left[\left(1-\frac{9 R_{i j}}{32 a}\right) \mathbf{I}+\frac{3 \mathbf{R}_{i j} \mathbf{R}_{i j}}{32 a R_{i j}}\right] \quad$ if $i \neq j$ and $R_{i j}<2 a$,

$C_{1}=1+\frac{2 a^{2}}{3 R_{i j}^{2}}$,

$C_{2}=1-\frac{2 a^{2}}{R_{i j}^{2}}$,

where $R_{i j} \equiv \sqrt{\mathbf{R}_{i j} \cdot \mathbf{R}_{i j}}$, and $\mathbf{R}_{i j} \equiv \mathbf{R}_{j}-\mathbf{R}_{i}$, where the vector $\mathbf{R}_{i}$ contains the three Cartesian coordinates of the position vector of the $i$ th bead. Parameter $a$ represents the bead hydrodynamic radius, $\zeta \equiv 6 \pi \eta a$ is the bead friction coefficient, and $\mathbf{I}$ is the $3 \times 3$ identity matrix. For the RPY tensor, the term $\partial / \partial \mathbf{R} \cdot \mathbf{D}$ appearing in Eq. (1) is zero. ${ }^{18}$ In the early work of Kirkwood and Riseman, ${ }^{19}$ and later in the Zimm ${ }^{20,21}$ theory, fluctuations of the equilibrium hydrodynamic interaction tensor were ignored in the interest of constructing a tractable analytical theory; $\mathbf{D}$ was taken to be its value with the structure fixed at equilibrium. A "free-draining" (FD) approximate model is obtained by neglecting hydrodynamic interactions $\left(\mathbf{D}_{i j}=0\right.$ for $\left.i \neq j\right)$. A "consistently averaged" 22 (CAHI) model is obtained by replacing $\mathbf{D}$ by its instantaneous ensemble average, $\langle\mathbf{D}\rangle$, in Eqs. 1 and 2. In this work, we will compare the "exact" (HI) model with the FD model. Although not presented in this work, simulations using the CAHI model were in good agreement with the HI model.

The connectivity between adjacent beads is described by means of a wormlike spring (WLS) model. Marko and Siggia $^{23,24}$ proposed a simple expression that matches the asymptotics of the exact wormlike chain model in both the small and large force limits, and fits the experimental DNA data of Smith et al. ${ }^{6}$ The result can be expressed as

$$
\mathbf{F}_{i j}^{s}=\frac{k_{B} T}{2 b_{k}}\left[\left(1-\frac{R_{i j}}{R_{o}}\right)^{-2}-1+\frac{4 R_{i j}}{R_{o}}\right] \frac{\mathbf{R}_{i j}}{R_{i j}},
$$

where $b_{k}$ is the Kuhn length of the molecule. Letting $N_{k}$ represent the number of Kuhn segments in the molecule and $N_{k, s}$ the number of Kuhn segments per spring, $R_{0}=N_{k, s} b_{k}$ is the maximum spring length, or the contour length of the portion of the molecule represented by one spring in our model. The contour length of the chain is then $L=N_{s} R_{0}$. This model has been used successfully in the literature. ${ }^{8-10,24,25}$

To complete the model, we now describe our treatment of the nonbonded interactions. From neutron scattering data ${ }^{26,27}$ for dilute solutions of linear polymer in a good solvent, one finds ideal chain behavior at small distances along the chain, and good solvent behavior at large distances. In this spirit, we consider the increase in energy due to the overlap of two submolecules (i.e., beads). Each submolecule is considered to be ideal with a (Gaussian) probability distribution with second moment $S_{s}^{2}=N_{k, s} b_{k}^{2} / 6$. Considering the energy penalty due to overlap of two Gaussian coils, ${ }^{21}$ one arrives at the following expression for the excluded volume potential between two beads of the chain:

$$
U_{i j}^{e v}=\frac{1}{2} v k_{B} T N_{k, s}^{2}\left(\frac{3}{4 \pi S_{s}^{2}}\right)^{3 / 2} \exp \left[\frac{-3 R_{i j}^{2}}{4 S_{s}^{2}}\right],
$$

where $v$ is the excluded volume parameter. Although this potential is not self-consistent (we have ignored any deformation of the coil caused by the overlap), Eq. (9) does provide the correct parameter dependence in the scaling regime. That is, for a specified $v$ we obtain good solvent scaling for static properties by either fixing $N_{s}$ and varying $N_{k, s}$, or by fixing $N_{k, s}$ and varying $N_{s}$. We demonstrate this in Sec. V D. Strictly speaking, the number of Kuhn segments per bead $\left(N_{k, b}\right)$, rather than $N_{k, s}$, should have been used in developing this excluded volume potential. However, $N_{k, s} / N_{k, b} \sim 1$ for most systems of interest.

Note that, as with most Brownian dynamics approaches to coarse-grained polymer dynamics, ours does not rigorously prevent chain-crossing. We make two comments regarding this point. First, Kumar and Larson ${ }^{28}$ have recently addressed the issue of chain-crossing with a model that, rather than imposing bead-bead repulsions, imposes "spring-spring" repulsions acting along the shortest line segment between nearby connector vectors. Nevertheless, with a soft potential, they find results essentially identical to those with bead-bead repulsions, while with a singular $\left(r^{-12}\right)$ potential the time step required becomes prohibitive. Second, we also performed simulations in which a harder inner core was also imposed in the form of Eq. (9) with $v_{\text {inner }}=2 v$ and $S_{s, \text { inner }}^{2}=S_{s}^{2} / 2$, and no change in chain properties was observed.

The Gaussian form of the excluded volume potential has been discussed by Öttinger ${ }^{22}$ in the context of renormalization group theory. Although other excluded volume potentials (hard-sphere, Lennard-Jones, soft exponential) have been used extensively in Brownian dynamics simulations, it is not obvious how the parameters in these models should vary with molecular discretization. This was the motivation for the development of Eq. (9), which explicitly contains the dependence on $N_{k, s}$. 


\section{PROPERTIES}

The measure of chain size most easily obtained from fluorescence microscopy experiments is the "stretch." The stretch of molecule $m, X_{m}$, is defined as the distance between the upstream-most portion of the molecule and the downstream-most portion,

$$
X_{m}=\max \left(\mathbf{R}_{i, x}\right)_{m}-\min \left(\mathbf{R}_{i, x}\right)_{m},
$$

and the ensemble average stretch is then simply

$$
\langle X\rangle=\frac{1}{N_{t}} \sum_{m=1}^{N_{t}} X_{m},
$$

where $N_{t}$ is the number of molecules, or trajectories, in our ensemble. The end-to-end distance, $R$, the radius-of-gyration tensor, $\mathbf{S}$, and the radius of gyration, $S$, are defined as usual

$$
\begin{aligned}
& R=\left\langle\operatorname{tr}\left(\left[\left(\mathbf{R}_{N_{b}}\right)-\left(\mathbf{R}_{1}\right)\right]\left[\left(\mathbf{R}_{N_{b}}\right)-\left(\mathbf{R}_{1}\right)\right]\right)^{1 / 2}\right\rangle, \\
& \mathbf{S}=\left\langle\frac{1}{N_{b}} \sum_{i=1}^{N_{b}}\left[\left(\mathbf{R}_{i}\right)-\left(\mathbf{r}_{c}\right)\right]\left[\left(\mathbf{R}_{i}\right)-\left(\mathbf{R}_{c}\right)\right]\right\rangle, \\
& S=\operatorname{tr}(\mathbf{S})^{1 / 2}
\end{aligned}
$$

where $\left(\mathbf{r}_{c}\right)$ is the center of mass of the molecule. The diffusivity tensor, $\mathbf{D}_{t r}$, and diffusivity, $D$, are given by

$$
\begin{aligned}
\mathbf{D}_{\mathrm{tr}}= & \lim _{n \rightarrow \infty} \frac{1}{2 \Delta t} \sum_{i=1}^{n}\left\langle\left[\mathbf{r}_{c}(i \Delta t)-\mathbf{r}_{c}([i-1] \Delta t)\right.\right. \\
& \left.-\mathbf{v}_{c}([i-1] \Delta t) \Delta t\right]\left[\mathbf{r}_{c}(i \Delta t)-\mathbf{r}_{c}([i-1] \Delta t)\right. \\
& \left.\left.-\mathbf{v}_{c}([i-1] \Delta t) \Delta t\right]\right\rangle, \\
D= & \frac{1}{3} \operatorname{tr}\left(\mathbf{D}_{\mathrm{tr}}\right)
\end{aligned}
$$

where $\mathbf{v}_{c}$ refers to the velocity of the solvent at the center of mass of the molecule.

In this work, the relaxation time was obtained by the same method as in the experiments ${ }^{5}$ from which we determined our model parameters. A stretched chain was allowed to relax to equilibrium. Near equilibrium (e.g., final $10 \%$ of decay), the longest relaxation time, $\lambda$, was determined from a fit to

$$
\left\langle X^{2}\right\rangle=A \exp \left(-\frac{t}{\lambda}\right)+B .
$$

Relaxation times determined from the decay of the equilibrium autocorrelation function for the end-to-end vector gave essentially identical results.

The microstructural contribution to the stress tensor, $\tau^{p}$ is given by

$$
\boldsymbol{\tau}^{p}=n \sum_{i=1}^{N_{b}}\left\langle\left(\mathbf{R}_{i}-\mathbf{r}_{c}\right) \mathbf{F}_{i}\right\rangle+n N_{s} k_{B} T \mathbf{I},
$$

with $n$ being the number density of molecules. ${ }^{20}$ In this work, we are interested in two types of flows: planar extension and simple shear. In planar extension, $\boldsymbol{\nabla} \mathbf{v}$ is given as

$$
(\boldsymbol{\nabla} \mathbf{v})_{p}=\left(\begin{array}{ccc}
\dot{\epsilon} & 0 & 0 \\
0 & -\dot{\epsilon} & 0 \\
0 & 0 & 0
\end{array}\right),
$$

while, in simple shear, we have

$$
(\boldsymbol{\nabla} \mathbf{v})_{s}=\left(\begin{array}{ccc}
0 & 0 & 0 \\
\dot{\gamma} & 0 & 0 \\
0 & 0 & 0
\end{array}\right) .
$$

Alternatively, one can represent the strength of the flow field through the Weissenberg number (We), which represents the ratio of the time scale of molecular relaxation to that of the solvent deformation. For planar extension and simple shear, we have $\mathrm{We}_{p} \equiv \dot{\epsilon} \tau$ and $\mathrm{We}_{s} \equiv \dot{\gamma} \tau$, respectively.

In the case of simple shear, the shear viscosity, $\eta$, and the first normal stress coefficient, $\Psi_{1}$, are obtained from $\tau^{p}$ and the shear rate $\dot{\gamma}$ by

$$
\begin{aligned}
& \eta=-\frac{\tau_{x y}^{p}}{\dot{\gamma}} \\
& \Psi_{1}=-\frac{\tau_{x x}^{p}-\tau_{y y}^{p}}{\dot{\gamma}^{2}} .
\end{aligned}
$$

The longest relaxation time obtained from the Rouse theory (i.e., Hookean springs, free-draining, theta solvent) is

$$
\lambda_{R}=\frac{\zeta b_{k}^{2} N_{k, s}}{24 k_{B} T \sin ^{2}\left(\frac{\pi}{2 N_{b}}\right)} .
$$

Throughout this work, we use the abbreviations HI and FD to refer to the non-free-draining and free-draining models, respectively.

\section{SIMULATION}

Equations (1)-(9) define our model for Brownian dynamics simulations of $\lambda$-phage DNA. The parameters appearing in the HI model are $\left\{L, N_{s}, b_{k}, a, v\right\}$. For YOYO-1 stained $\lambda$-phage DNA we have $L=21 \mu \mathrm{m} .^{5}$ The parameter $N_{s}$ defines the level of discretization of the molecule, and we choose $N_{s}=10$ for $21 \mu \mathrm{m} \lambda$-phage DNA. The remaining three parameters $\left(b_{k}, a, v\right)$ were chosen so that our model reproduces available experimental data. For YOYO-1 stained $21 \mu \mathrm{m} \lambda$-phage DNA in a $43.3 \mathrm{cP}$ solvent at $23 \mathrm{C}$, we have available experimental values ${ }^{5}$ of the equilibrium stretch $(1.5$ $\mu \mathrm{m})$, and relaxation time $(4.1 \mathrm{~s})$. We also have available diffusivity data for native DNA fragments $(<1 \mu \mathrm{m})$ obtained from light-scattering data ${ }^{29}$ and diffusivity data for TOTO-1 stained fragmented and concatenated $\lambda$-phage DNA (2-140 $\mu \mathrm{m})$ in a $0.95 \mathrm{cP}$ solvent. ${ }^{3}$ The diffusivity data for the stained DNA can be scaled to correspond to native DNA in an solvent of arbitrary viscosity, $\eta$, by the scaling $D \sim \eta^{-1}$, and correcting for the change in persistence length due to dye intercalation. Smith et al. ${ }^{3}$ found that the diffusivity of TOTO-1 stained DNA needed to be scaled by $(1.75)^{2 / 5}$ to 
bring it into line with the native DNA fragments of Sorlie and Pecora. ${ }^{29}$ They also reported that this correction factor decreased to $(1.32)^{2 / 5}$ when YOYO-1 stain was used. ${ }^{5}$ Using the data of Smith et al., ${ }^{3}$ we can therefore estimate the diffusivity of YOYO-1 stained $21 \mu \mathrm{m} \lambda$-phage DNA in a 43.3 cP solvent at $23 \mathrm{C}$ to be $0.0115 \mu \mathrm{m}^{2} / \mathrm{s}$.

The parameters $\left\{b_{k}, a, v\right\}$ were then determined so that the model reproduced experimental values of the equilibrium stretch $(1.5 \mu \mathrm{m})$, relaxation time $(\lambda=4.1 \mathrm{~s})$, and the estimated diffusivity $\left(0.0115 \mu \mathrm{m}^{2} / \mathrm{s}\right)$. This gave $\left\{b_{k}\right.$ $\left.=0.106 \mu \mathrm{m}, a=0.077 \mu \mathrm{m}, v=0.0012 \mu \mathrm{m}^{3}\right\}$. This value of $a$ corresponds to a bead diffusivity of $k_{B} T / \zeta_{\mathrm{HI}}$ $=0.065 \mu \mathrm{m}^{2} / \mathrm{s}$, and hydrodynamic interaction parameter, ${ }^{20}$ $h^{*}=0.16$. The relaxation time was determined in the same way as was done in the experiment; ${ }^{5}$ a fully stretched chain was allowed to relax to equilibrium, and the tail of the curve was fit to Eq. (17). We note here that Smith et al. ${ }^{3}$ reasoned, using the scaling $D \sim b_{k}^{-2 / 5} L^{-3 / 5}$, that the Kuhn length of TOTO-1 stained DNA increased by a factor of 1.75 relative to the native DNA value of $b_{k}=0.106 \mu \mathrm{m}$, while, for YOYO-1 stained DNA, the Kuhn length increased by a factor of 1.32. In our simulations, however, we found that that we needed $b_{k}=0.106 \mu \mathrm{m}$ (the native DNA value, by coincidence) to reproduce both the experimental relaxation and diffusivity of YOYO-1 stained DNA.

For the FD model, we again used $v=0.0012$, and $b_{k}$ $=0.106 \mu \mathrm{m}$, which left the simulation equilibrium stretch unchanged. The free-draining friction coefficient was chosen so that the FD model reproduced the experimental relaxation time $(\lambda=4.1 \mathrm{~s})$. This resulted in a bead diffusivity of $k_{B} T / \zeta_{\mathrm{FD}}=0.084 \mu \mathrm{m}^{2} / \mathrm{s}$, indicating a $23 \%$ decrease in the bead friction coefficient relative to the HI model. This is consistent with previous findings. ${ }^{30}$

A first order semi-implicit scheme described in an earlier paper $^{16}$ was used to integrate Eq. (1). A time step of $0.1 \min \left\{\zeta S_{s}^{2} / k_{B} T,\left[(\nabla \mathbf{v}):(\nabla \mathbf{v})^{T}\right]^{-1 / 2}\right\}$ was used in all simulations. The quantity $\zeta S_{s}^{2} / k_{B} T$ represents the time scale for diffusion of the submolecule, while $\left[(\boldsymbol{\nabla} \mathbf{v}):(\boldsymbol{\nabla} \mathbf{v})^{T}\right]^{-1 / 2}$ represents the flow time scale. The decomposition of the diffusion tensor [Eq. (2)] was performed using Fixman's method $^{31}$ as described in earlier work. ${ }^{16}$ Here we simply note that Fixman's method involves a fast polynomial approximation to the square root of the diffusion tensor, and we required $\sim 5 \%$ relative accuracy in the decomposition for all simulations. A method for accurately determining the error in the decomposition is discussed in detail in our earlier work. ${ }^{16}$

We performed both HI and FD simulations for chains of length $21 \mu \mathrm{m}\left(N_{s}=10\right), 42 \mu \mathrm{m}\left(N_{s}=20\right), 84 \mu \mathrm{m}\left(N_{s}\right.$ $=40)$, and $126 \mu \mathrm{m}\left(N_{s}=60\right)$ in extensional and shear flow. In all computations except those in Sec. V D, the level of molecular discretization $\left(N_{k, s}=19.8\right)$ was kept constant as the molecular weight was increased. In Sec. V D, both $N_{k}$ and $N_{k, s}$ are varied to determine the effect of molecular discretization on model predictions.

Most of our results are presented in terms of Weissenberg numbers. These Weissenberg numbers are based on the relaxation time, $\lambda$, of the model in question ( $\mathrm{HI}$ or $\mathrm{FD}$ ). Therefore, a comparison at equal Weissenberg numbers does not indicate equivalent strain rates at a given molecular

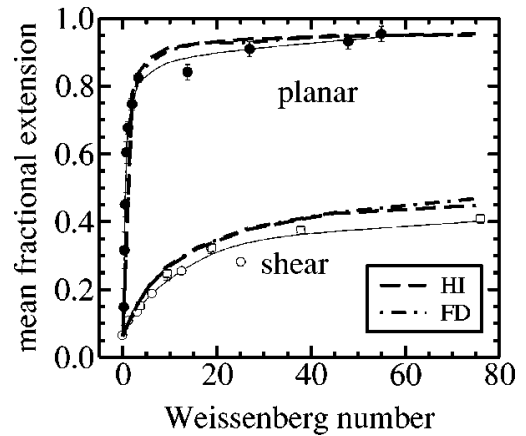

FIG. 1. Mean fractional stretch vs Weissenberg number. Our simulation results for the HI (dashed lines) and FD (dotted-dashed lines) models superimposed on the experimental data of Smith, Babcock, and Chu (Ref. 2). The solid lines were provided with the experimental data and serve only to guide the eye.

weight, with the exception of the $21 \mu \mathrm{m}$ chains. For longer chains, at a given Weissenberg number, the FD strain rate is always less than that of the HI case.

\section{RESULTS AND DISCUSSION}

\section{A. Comparison to experiment: $\lambda$-phage DNA}

Figure 1 shows the mean steady-state fractional extension versus Weissenberg number from experiment ${ }^{2}$ and our simulations. Results are shown for $21 \mu \mathrm{m}$ DNA in both shear and planar extension. We find good agreement between our simulations and experiment. At this molecular weight, the HI and FD models give practically identical results for steady-
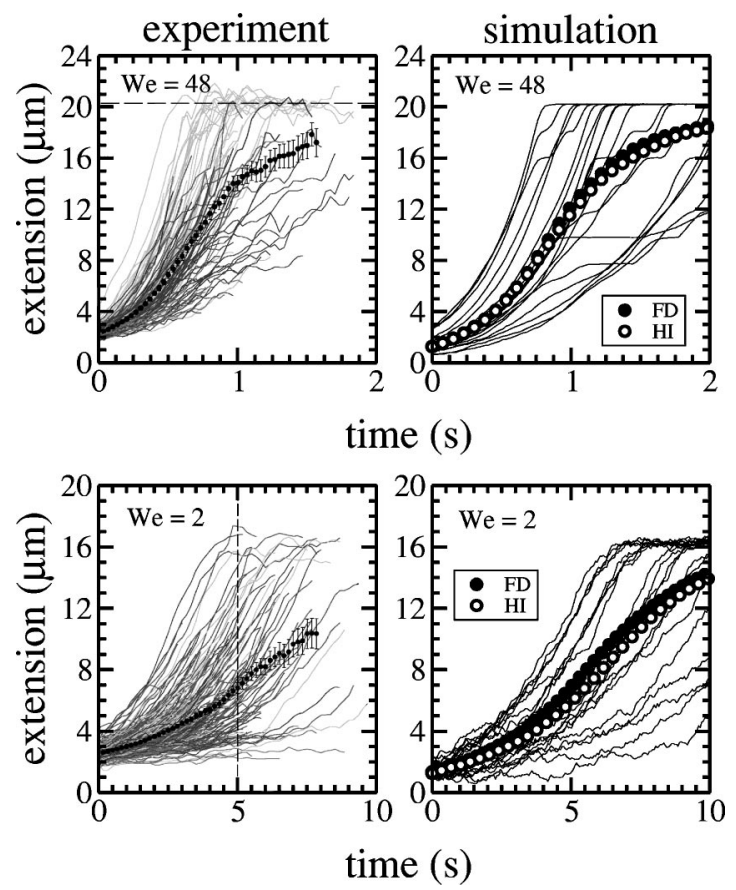

FIG. 2. Mean stretch vs time. Comparison of our simulations with the experimental data of Smith and Chu (Ref. 5). The data points are ensemble averages, while the thin lines correspond to individual molecular trajectories (in the simulations, these are HI trajectories). All simulations were started from random equilibrium configurations. 


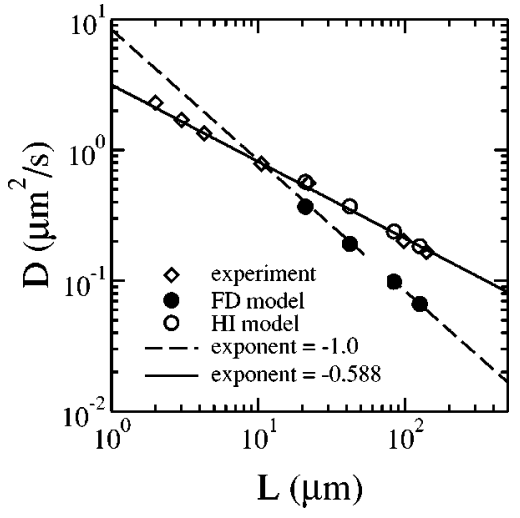

FIG. 3. Diffusivity as a function of molecular weight for the FD and HI models. Experimental data is from Smith, Perkins, and Chu (Ref. 3). All data has been scaled to native DNA in $1 \mathrm{cP}$ solvent (see text). The solid and dashed lines are the theoretical scalings for the HI and FD models, respectively.

state stretch. As molecular weight is increased, discrepancies appear between the two models. These discrepancies are discussed in Sec. V B.

Figure 2 shows the transient mean fractional stretch from experiment ${ }^{5}$ and our simulations, plotted in real time. Results are shown for startup of planar extension at $\mathrm{We}=2$ and $\mathrm{We}$ $=48$. In the experimental plot, the thin lines are individual molecular trajectories, while the symbols are the mean fractional extension at the indicated point in time. A few individual trajectories are also shown from the simulations. As has been noted in the literature, ${ }^{9,10}$ the average stretch does not appear to be significantly affected by neglecting hydrodynamic interactions, provided the friction coefficient is fit to the experimental relaxation time. In the $\mathrm{We}=48$ simulations (top right in Fig. 2), transient plateaus appear in some molecular trajectories. As discussed by Smith and $\mathrm{Chu}^{5}$ and Larson et al., ${ }^{10}$ these plateaus occur when molecules form folded configurations during the unraveling process.

Figure 3 shows the equilibrium scalar diffusivity, $D$, as a function of molecular weight. Results are shown from the experiments of Smith et al., ${ }^{3}$ as well as our simulations. All data have been scaled to a solvent viscosity of $1 \mathrm{cP}$, and adjusted (as described in Sec. IV) for dye intercalation to give native DNA results. As expected, the molecular weight scaling of the HI model matches that of experiment, while the FD model does not. Also shown are the predicted scaling exponents from the Rouse $\left(D \sim L^{-1}\right)$ and Zimm ( $D$ $\sim L^{-0.588}$ ) theories. Zimm scaling is observed in the experimental data for DNA starting at around $3 \mu \mathrm{m}$. As seen in Fig. 3 , all of our HI simulation results follow Zimm scaling.

\section{B. Predictions for longer chains}

In this section, we predict and examine various dynamic and static properties of DNA for a range of molecular weights. Having fit both the HI and FD models to the same experimental data for $21 \mu \mathrm{m}$ DNA, we can quantify the error in these properties that arises when hydrodynamic interactions are ignored.

Figure 4 shows the mean steady-state fractional stretch for $126 \mu \mathrm{m}$ DNA. Ignoring hydrodynamic interactions leads

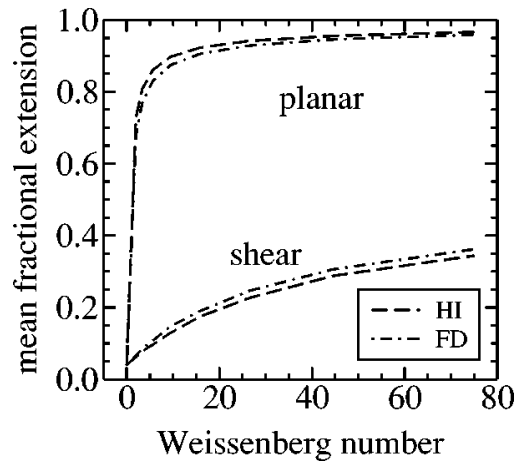

FIG. 4. Mean steady-state fractional stretch vs Weissenberg number. Simulation results for $126 \mu \mathrm{m}$ DNA in planar extension and simple shear.

to a slightly lower stretch for planar extension, and a higher stretch in simple shear. This is expected: in simple shear, hydrodynamic interactions attenuate the relative motion of the beads, thereby damping the dynamic tumbling motion of the chain (which involves beads sliding past one another). The same trend was also found by Petera and Muthukumar ${ }^{32}$ for bead-rod chains. In extensional flow, this tumbling is absent. Recall that we determined the bead friction coefficient so that the simulation relaxation time matched that of the actual chain. The result was that the bead friction coefficient in the FD model was $23 \%$ less than that of the HI model. When the chain is stretched, hydrodynamic interactions are diminished as the beads become further separated, and the chain becomes "more free-draining." Therefore, for planar extension the steady-state stretch is higher when we include hydrodynamic interactions $\left(\zeta_{\mathrm{HI}}>\zeta_{\mathrm{FD}}\right)$.

Figure 5 shows the transient mean fractional stretch as a function of real time for $21 \mu \mathrm{m}$ and $126 \mu \mathrm{m}$ DNA, respectively, in planar extension. As molecular weight increases, the calculated approach to steady state for the FD model becomes retarded due to its inability to correctly capture the relaxation time.

In a FD model, the diffusivity tensor, $\mathbf{D}_{\mathrm{tr}}$, is isotropic and independent of molecular configuration. In reality, due to hydrodynamic interactions, $\mathbf{D}_{\mathrm{tr}}$ is anisotropic and dependent on the molecular configuration. Figure 6 illustrates the anisotropic diffusivity of $21 \mu \mathrm{m}$ and $126 \mu \mathrm{m}$ DNA molecules in planar extension and simple shear. Steady-state values of the diagonal components of $\mathbf{D}_{\mathrm{tr}}^{\mathrm{HI}}$ are shown as a function of
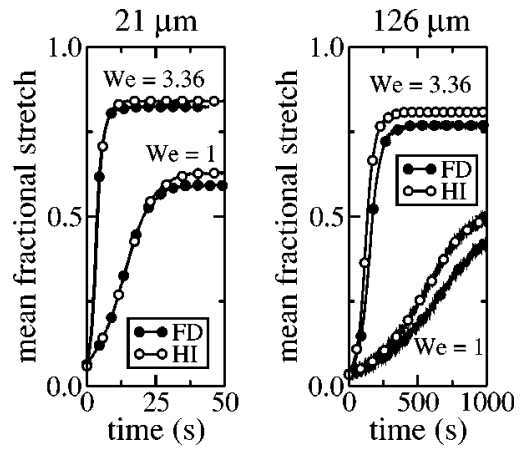

FIG. 5. Mean fractional stretch vs time. Simulation results for $21 \mu \mathrm{m}$ and $126 \mu \mathrm{m}$ DNA in planar extension. 


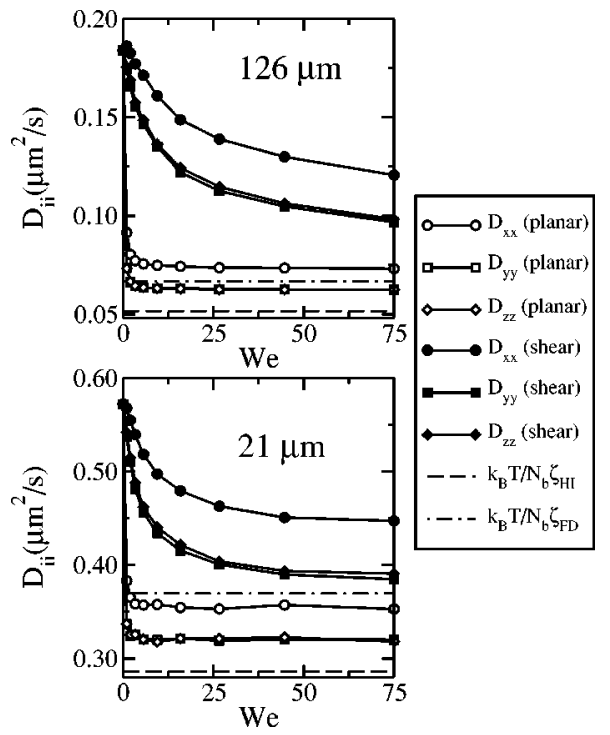

FIG. 6. Diagonal components of the diffusivity tensor for the $\mathrm{HI}$ model as a function of Weissenberg number. Simulation results for $21 \mu \mathrm{m}$ DNA and $126 \mu \mathrm{m}$ DNA. Both planar extension (open symbols) and simple shear (solid symbols) are shown. The dashed lines correspond to the "freedraining" diffusivities of the HI and FD models. All data have been scaled to correspond to native DNA in a $1 \mathrm{cP}$ solvent (as described in Sec. IV).

Weissenberg number. Also shown are the "free-draining" diffusivities of the HI and FD models. For the HI model, this asymptotic limit is never reached; these finitely extensible chains cannot achieve the bead-bead separation necessary to approach $D^{\mathrm{HI}}=k_{B} T / N_{b} \zeta_{\mathrm{HI}}$. The free-draining model, having always $D^{\mathrm{FD}}=k_{B} T / N_{b} \zeta_{\mathrm{FD}}$, is, of course, unable to capture the highly anisotropic behavior.

\section{Weissenberg number scaling of shear rheology}

To examine the dependence of $\eta$ and $\Psi_{1}$ on Weissenberg number, we performed simulations with $N_{s}=40$ over a wide range of Weissenberg numbers. Both the HI and FD models were investigated. The results are shown in Fig. 7, along with the transverse component of the gyration tensor, $S_{y y}$. In these runs, the Weissenberg numbers are based on the longest Rouse relaxation time, as we are here only concerned with scaling laws. The parameters used in the simulations presented in this section (and in Sec. VD) were $b_{k}$ $=0.14 \mu \mathrm{m}, v=0.0027 \mu \mathrm{m}^{3}$, and $a=0.138 \mu \mathrm{m}$.

The results in Fig. 7(A) suggest the existence of two distinct scaling regimes in the shear rheology of chains in flow. In this section, we analyze these results in the light of relatively simple scaling arguments. ${ }^{33,34}$ A steady-state balance between entropic and hydrodynamic forces leads to an expression for the $x$-component of the tensile force in a molecule in shear flow,

$$
F_{x} \sim \zeta \dot{\gamma} \delta_{y},
$$

where $\delta_{y}$ represents the length scale of chain fluctuations transverse to the flow direction. Examination of the polymer contribution to the stress tensor at high Weissenberg number, assuming that the characteristic chain length in the flow direction is roughly constant (at some fraction of the contour length), gives
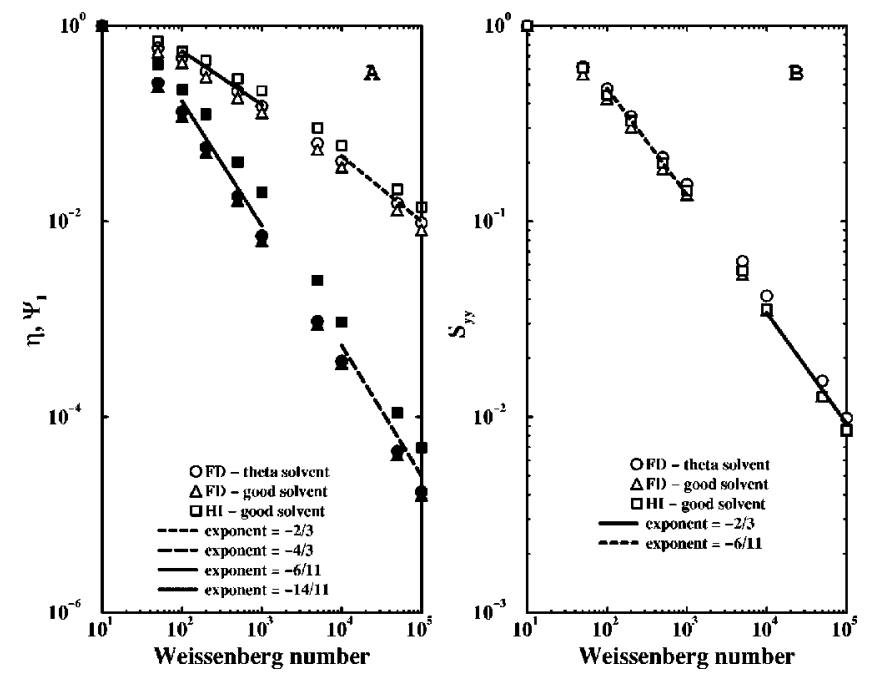

FIG. 7. (A) The shear viscosity (open symbols) and first normal stress coefficient (solid symbols) vs Weissenberg number for various models of $84 \mu m$ DNA. (B) The $y y$-component of the gyration tensor versus Weissenberg number for the various models. In both (A) and (B), the Weissenberg numbers are based on the Rouse relaxation time, and properties are reduced by their $\mathrm{We}=10$ values. Local scaling exponents are shown for two regions of the curves.

$$
\begin{aligned}
& \eta \sim \delta_{y}^{2}, \\
& \Psi_{1} \sim \delta_{y} / \dot{\gamma} .
\end{aligned}
$$

Taking $\delta_{y}$ to be proportional to the transverse distance a chain end diffuses during one flip of the chain, a balance of streamwise convective transport $\dot{\gamma} \delta_{y}$ and transverse diffusive transport $D / \delta_{y}^{2}$, with $D$ being the (constant) bead diffusivity, leads to

$$
\delta_{y} \sim \dot{\gamma}^{-1 / 3}
$$

This is essentially the classical Graetz-Leveque result for the thickness of a concentration boundary layer in shear flow. ${ }^{35,33}$ Using the result in the expressions for the viscometric properties gives that $\eta \sim \mathrm{We}^{-2 / 3}, \Psi_{1} \sim \mathrm{We}^{-4 / 3}$, in good agreement with the results in Fig. 7(A) for We $\gtrsim 10^{4}$. In this regime, the computed value of $S_{y y}$ is also in agreement with this simple argument: $S_{y y} \sim \delta_{y}^{2} \sim \mathrm{We}^{-2 / 3}$. These results are also recovered for a wide variety of simple dumbbell or chain based constitutive equations (e.g., the FENE-P and FENE-PM), essentially because of the generic nature of the Graetz-Leveque argument made above. ${ }^{36}$

At intermediate shear rates, $10^{2} \lesssim \mathrm{We} \lesssim 10^{3}$, the simulations do not follow the above simple scaling, but rather give exponents close to $-1 / 2$ and $-5 / 4$. Similar scalings were observed by Doyle et al. ${ }^{33}$ for simulations of bead-rod chains. They argued that these "non-classical" exponents could be explained by arguments based on anomalous diffusion. In chain relaxation simulations, they found that $\delta_{y}^{2}$ $\sim t^{\alpha}$, with $\alpha \approx 3 / 4$. This subdiffusive response is related to the connectivity constraint, ${ }^{34}$ and translates into a diffusivity that depends on position as $D \sim \delta_{y}^{-2 / 3}$. Substituting this relation into the flux balance above gives that $\delta_{y} \sim \dot{\gamma}^{-3 / 11}$, yielding $S_{y y} \sim \mathrm{We}^{-6 / 11}, \eta \sim \mathrm{We}^{-6 / 11}$ and $\Psi_{1} \sim \mathrm{We}^{-14 / 11}$. The corresponding slopes are shown on Fig. 7 and are seen to be in 

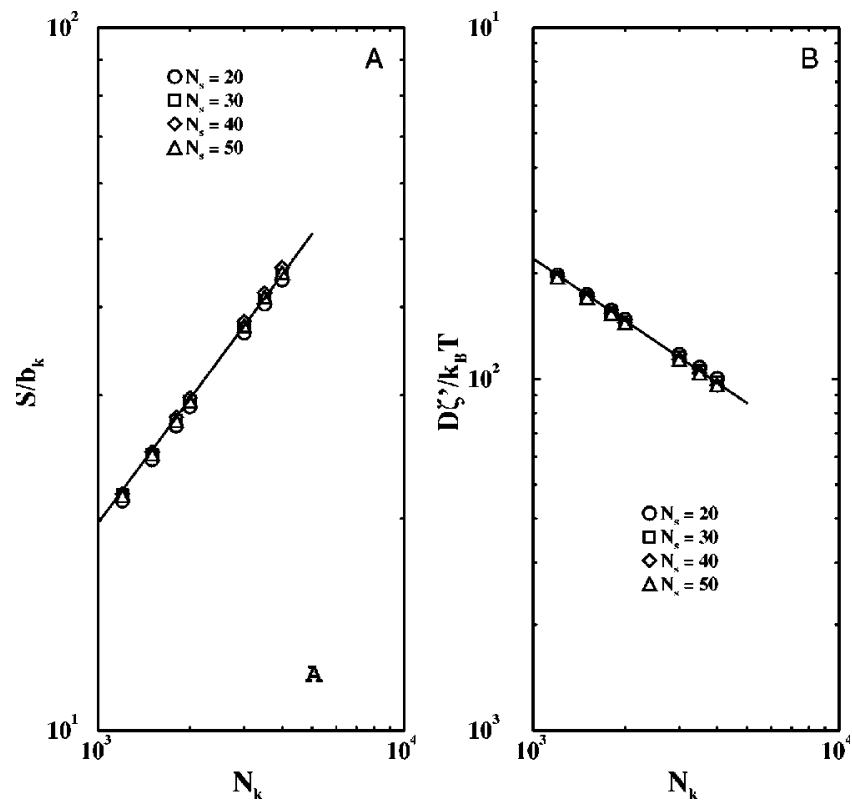

FIG. 8. Molecular weight dependence of (A) the radius of gyration and (B) the diffusivity for the HI model at various levels of molecular discretization $\left(N_{s}\right)$. The solid lines correspond to the theoretical scalings, $S \propto N_{k}^{0.588}$ and $D \propto N_{k}^{-0.588}$.

good agreement with the simulation results. At very high shear rates, Doyle et al. observed a leveling off of $\eta$, when $\delta_{y}$ approaches the (fixed) bond length. As described above, we see no such leveling off, but rather a reversion to a classical result. This can be explained by the fact that, in contrast to the bead-rod chains, here there is no bond length constraint to fix a small length scale.

\section{Parameter dependence}

In Sec. II we stated that the excluded volume potential given by Eq. (9) should give the correct parameter dependence in the scaling regime. To illustrate this point, we performed equilibrium simulations for a range of molecular weights $\left(N_{k}\right)$ and molecular discretizations $\left(N_{k, s}\right)$. In the simulations presented in this section, we used $b_{k}=0.14$ $\mu \mathrm{m}$, and $v=b_{k}^{3}=0.0027 \mu \mathrm{m}^{3}$. We also let each submolecule (spring) obey Zimm scaling, $a=C_{a} b_{k}\left(N_{k, s} / 6\right)^{1 / 2}$, giving $\zeta=6 \pi \eta a=\zeta^{\prime} N_{k, s}^{1 / 2}$. The constant of proportionality was taken to be $C_{a}=0.64$.

Figure 8(A) shows the molecular weight dependence of $S$ for different $N_{s}$. Our simple excluded volume potential clearly contains the correct dependence on $N_{k, s}$. Once $v$ is specified, $N_{s}$ can be changed at constant $N_{k}$, and no significant change in $S$ is observed. Molecular weight can be increased by increasing $N_{k}$ at constant $N_{k, s}$ or $N_{s}$, and the correct scaling is observed. Also shown in Fig. 8(A) is the theoretical good-solvent scaling.

Figure 8(B) shows the molecular weight dependence of $D \zeta^{\prime} / k_{B} T$ for different $N_{s}$. We find that at fixed $N_{s}$ we can increase molecular weight and obtain the correct theoretical scaling. We already demonstrated in Fig. 3 that we can obtain the correct scaling by fixing $N_{k, s}$ and increasing molecular weight. Also, increasing $N_{s}$ at constant molecular weight does not significantly affect the diffusivity.
To summarize, if we assume ideal, nondraining behavior at the spring (submolecule) level, the HI model presented in this work provides the correct dependence on molecular discretization (for equilibrium properties). That is, once $\left\{b_{k}\right.$, $\left.a^{\prime}, v\right\}$ have been determined, molecular weight can be changed by varying $N_{k, s}$ or $N_{s}$, with static properties and diffusivity scaling correctly with molecular weight. Also, $N_{s}$ can be changed at constant molecular weight (and constant $\left.\left\{v, k_{B} T / \zeta^{\prime}, a^{\prime}\right\}\right)$ with very little change in static properties or diffusivity.

\section{CONCLUSIONS}

We have presented a fully parametrized nondraining (HI) bead-spring model for DNA with parameters determined from experimental data for $21 \mu \mathrm{m}$ DNA, and demonstrated that the model predictions are in quantitative agreement with available experimental data. In addition, we examined a freedraining (FD) model in which hydrodynamic interactions are ignored. In order to obtain equivalent relaxation times at the experimental molecular weight, the FD bead friction coefficient needed to be $23 \%$ lower than that of the HI model. Both models adequately predict the configurational properties of $21 \mu \mathrm{m}$ DNA in simple shear and planar extension. At higher molecular weights, however, the approach to steady state for the FD model lags behind that of the HI model. This lag increases considerably with increasing molecular weight, and is mainly due to the inability of the free-draining relaxation time to scale properly with molecular weight. By comparison to experimental data, we have shown that the HI model correctly predicts the diffusivity of longer DNA, while the FD model does not.

The high Weissenberg number scaling behavior of $\eta$ and $\Psi_{1}$ is independent of solvent quality and hydrodynamic interactions. For $\mathrm{We} \gtrsim 10^{4}, \eta$ and $\Psi_{1}$ scale as $\mathrm{We}^{-2 / 3}$ and $\mathrm{We}^{-4 / 3}$, respectively, consistent with classical arguments balancing convection and diffusion of chain ends. For $10^{2} \leq \mathrm{We} \leq 10^{3}$, these properties scale as $\mathrm{We}^{-6 / 11}$ and $\mathrm{We}^{-14 / 11}$, consistent with anomalously slow diffusion due to the connectivity constraint.

For the HI model, all molecular weight scalings are in good agreement with expected values. Using consistently averaged hydrodynamic interactions gives static and dynamic results in quantitative agreement with fluctuating hydrodynamics. The excluded volume potential presented in this work provides the correct dependence on molecular discretization (Kuhn segments per spring): i.e., at fixed molecular weight, a change in molecular discretization does not significantly affect static properties, and good solvent scaling is obtained by either varying the number of springs at constant molecular discretization, or by varying the molecular discretization at constant number of beads.

\section{ACKNOWLEDGMENTS}

Acknowledgment of support is made to the National Science Foundation (EEC-0085560), the Defense Advanced Research Projects Agency, and to the donors of the Petroleum Research Fund, administered by the American Chemical So- 
ciety. The authors also thank Professor Ronald G. Larson and Professor Eric S. G. Shaqfeh for helpful discussions.

${ }^{1}$ J. Han and H. G. Craighead, Science 288, 1026 (2000).

${ }^{2}$ D. E. Smith, H. P. Babcock, and S. Chu, Science 283, 1724 (1999).

${ }^{3}$ D. E. Smith, T. T. Perkins, and S. Chu, Macromolecules 29, 1372 (1996).

${ }^{4}$ S. B. Smith, L. Finzi, and C. Bustamante, Science 258, 1122 (1992).

${ }^{5}$ D. E. Smith and S. Chu, Science 281, 1335 (1998)

${ }^{6}$ C. Bustamante, J. F. Marko, E. D. Siggia, and S. Smith, Science 265, 1600 (1994).

${ }^{7}$ T. T. Perkins, D. E. Smith, and S. Chu, Science 276, 2016 (1997).

${ }^{8}$ R. G. Larson, T. T. Perkins, D. E. Smith, and S. Chu, Phys. Rev. E 55, 1794 (1997).

${ }^{9}$ J. S. Hur, E. S. G. Shaqfeh, and R. G. Larson, J. Rheol. 44, 713 (2000).

${ }^{10}$ R. G. Larson, H. Hu, D. E. Smith, and S. Chu, J. Rheol. 43, 267 (1999).

${ }^{11}$ J. S. Hur, E. S. G. Shaqfeh, H. P. Babcock, D. E. Smith, and S. Chu, J. Rheol. 45, 421 (2001).

${ }^{12}$ H. Jian, A. V. Vologodskii, and T. Schlick, J. Comput. Phys. 136, 168 (1997).

${ }^{13}$ H. Jian, T. Schlick, and A. Vologodskii, J. Mol. Biol. 284, 287 (1998).

${ }^{14}$ T. Schlick, D. A. Beard, J. Huang, D. A. Strahs, and X. Qian, Comput. Sci. Eng. 2, 38 (2000).

${ }^{15}$ R. M. Jendrejack, J. J. de Pablo, and M. D. Graham, to appear in "Technical Proceedings of the 2002 International Conference on Modeling and Simulation of Microsystems" (Applied Computational Research Society, Cambridge, 2002).

${ }^{16}$ R. M. Jendrejack, M. D. Graham, and J. J. de Pablo, J. Chem. Phys. 113, 2894 (2000).
${ }^{17}$ J. Rotne and S. Prager, J. Chem. Phys. 50, 4831 (1969).

${ }^{18}$ In a previous paper (Ref. 16), we also used the RPY tensor, but omitted the divergence term without comment.

${ }^{19}$ J. G. Kirkwood and J. Riseman, J. Chem. Phys. 16, 565 (1948).

${ }^{20}$ R. B. Bird, C. F. Curtiss, R. C. Armstrong, and O. Hassager, Dynamics of Polymeric Liquids (Wiley, New York, 1987), Vol. 2.

${ }^{21}$ M. Doi and S. F. Edwards, The Theory of Polymer Dynamics (Clarendon, Oxford, 1986).

${ }^{22}$ H.-C. Öttinger, Stochastic Processes in Polymeric Fluids (Springer, Berlin, 1996).

${ }^{23}$ J. F. Marko and E. D. Siggia, Macromolecules 27, 981 (1994).

${ }^{24}$ J. F. Marko and E. D. Siggia, Macromolecules 28, 8759 (1995).

${ }^{25}$ B. Ladoux and P. S. Doyle, Europhys. Lett. 52, 511 (2000).

${ }^{26}$ G. R. Strobl The Physics of Polymers (Springer, Berlin, 1997).

${ }^{27}$ B. Farnoux, F. Bou, J. P. Cotton, M. Daoud, G. Jannink, M. Nierlich, and P. G. de Gennes, J. Phys. (Paris) 39, 77 (1978).

${ }^{28}$ S. Kumar and R. G. Larson, J. Chem. Phys. 114, 6937 (2001).

${ }^{29}$ S. Sorlie and R. Pecora, Macromolecules 23, 487 (1990).

${ }^{30}$ J. W. Hatfield and S. R. Quake, Phys. Rev. Lett. 82, 3548 (1999).

${ }^{31}$ M. Fixman, Macromolecules 19, 1204 (1986).

${ }^{32}$ D. Petera and M. Muthukumar, J. Chem. Phys. 111, 7614 (1999).

${ }^{33}$ P. S. Doyle, E. S. G. Shaqfeh, and A. P. Gast, J. Fluid Mech. 334, 251 (1997).

${ }^{34}$ P. Grassia and E. J. Hinch, J. Fluid Mech. 308, 255 (1996).

${ }^{35}$ W. M. Deen, Analysis of Transport Phenomena (Oxford, New York, 1998).

${ }^{36}$ L. E. Wedgewood, D. N. Ostrov, and R. B. Bird, J. Non-Newtonian Fluid Mech. 40, 119 (1991). 